\title{
TRISTAR - a tracer in situ TDLAS for atmospheric research
}

\author{
F.G. Wienhold ${ }^{1, *}$, H. Fischer ${ }^{1, * *}$, P. Hoor ${ }^{1}$, V. Wagner ${ }^{1}$, R. Königstedt ${ }^{1}$, G.W. Harris ${ }^{2}$, J. Anders ${ }^{3}$, R. Grisar ${ }^{3}$, M. Knothe ${ }^{3}$, \\ W.J. Riedel ${ }^{3}$, F.-J. Lüibken ${ }^{4}$, T. Schilling ${ }^{4}$
}

\begin{abstract}
A novel tunable diode laser absorption spectrometer (TDLAS) called TRISTAR (tracer in situ TDLAS for atmospheric research) has been developed for airborne tracegas measurements in the upper troposphere and lower stratosphere. Up to three different species can be measured simultaneously with high temporal resolution $(<1 \mathrm{~s})$ using up to three individual lead-salt diode lasers. The lasers are operated in a time-multiplexed mode using a novel modulation scheme that combines laser operation in a pulsed-current mode with a combination of rapid scanning and two-tone frequency modulation. The latter improves the signal-to-noise ratio of phase-sensitive detection when compared to standard lockin techniques because of the reduction of instrument noise at higher detection frequencies. TRISTAR has been used in twochannel mode to measure $\mathrm{CO}$ and $\mathrm{N}_{2} \mathrm{O}$ during two airborne polar stratospheric campaigns in January and March 1997. These species were detected using integration periods of $1 \mathrm{~s}$ with a precision of $\pm 2 \%(3 \sigma)$ and a calibration accuracy of $\pm 2.8 \%$ during a total of 11 measurement flights up to a maximum altitude of $12.5 \mathrm{~km}$. More recently all three channels have been operated simultaneously for $\mathrm{CO}, \mathrm{CH}_{4}$, and $\mathrm{N}_{2} \mathrm{O}$ with comparable results.
\end{abstract}

PACS: $39.90 .+\mathrm{w} ; 93.85 .+\mathrm{q} ; 94.10 . \mathrm{Fa}$

In recent years the technique of tunable diode laser absorption spectroscopy (TDLAS) has found widespread application in the field of atmospheric research [1]. The technique is based on recording of individual rovibrational absorption lines of the target molecules in the mid-infrared spectral region and exploits the high spectral brightness and narrow tunable emission bandwidth of lead-salt diode lasers. By pumping the atmospheric sample rapidly through a low-pressure cell (approximately 50 mbar), the width of the pressure-broadened absorption line is reduced and overlap with other absorptions in air is minimized, resulting in excellent specificity. In addition, the sensitivity is enhanced by passing the laser beam through a multi-pass reflection cell, yielding sub-ppbv detection limits for many small molecules of atmospheric relevance, for example, $\mathrm{HCHO} . \mathrm{H}_{2} \mathrm{O}_{2}, \mathrm{NO}_{2}, \mathrm{HNO}_{3}, \mathrm{NH}_{3}, \mathrm{OCS}$, $\mathrm{HCl} . \mathrm{C}_{2} \mathrm{H}_{2}, \mathrm{CO}, \mathrm{CH}_{4}$, and $\mathrm{N}_{2} \mathrm{O}$. TDLAS systems have been successfully applied for trace-gas detection throughout the troposphere and lower stratosphere on ground-based $[2,3]$, shipboard [4], and airborne platforms [2,5-8]. Specialized instruments have been adapted for measurement of trace-gas fluxes using micrometeorological techniques $[9,10]$ and for the measurement of the stable isotopomers in methane [11].

Here we report on a newly developed computer-controlled, multi-laser tracer in situ TDLAS for atmospheric research (TRISTAR) which is optimized for high-precision (\% range) air-borne trace-gas measurements with high temporal resolution $(1 \mathrm{~Hz})$ in the tropopause region. In Sect. 1 the opticalmechanical setup of the instrument is described, and the electronic components (high-frequency modulation and detection scheme, instrument control, data acquisition) are described in Sect. 2. An inlet configuration which allows in situ calibrations during the flights is described in Sect. 3. Examples of trace-gas measurements obtained during three Arctic winter campaigns in 1997 and 1998 are presented in Sect. 4, together with a description of the data evaluation and quality control procedures.

\section{Optical and mechanical setup}

Operation of a TDLAS system, especially on an airborne platform, requires careful design to meet the stringent demands of vibration isolation, shock resistance, and weight minimization. This affects in particular the optical and mechanical setup of the system. For TRISTAR a very rigid optical layout was developed at the Fraunhofer Institut für Physikalische Meßtechnik (IPM). It consists of a $60 \mathrm{~cm} \times 45 \mathrm{~cm} \times 3 \mathrm{~cm}$ aluminum board recessed for weight reduction, a single liquidnitrogen $\left(\mathrm{LN}_{2}\right)$ cryostat, housing three lasers and two detec- 
tors, a multi-pass cell based on White's design [12], and a set of ellipsoidal, parabolic, and plane mirrors for collimation, steering, and re-focussing of the optical beam (Fig. 1).

The newly developed liquid-nitrogen cryostat relies on a novel evaporation principle which provides adjustable cooling power, essentially independent of the operating temperature [13]. Cooling of the individual cold stations is accomplished via small tubes to a heat exchanger inside the cold fingers, where the $\mathrm{LN}_{2}$ evaporates, with the gaseous $\mathrm{N}_{2}$ returning via tubes to the $\mathrm{LN}_{2}$ reservoir through a critical orifice. The $\mathrm{LN}_{2}$ flow is driven by the pressure difference between the height of the orifice and the $\mathrm{LN}_{2}$ level inside the heat exchanger. The diameter of the orifice limits the mass flow of $\mathrm{N}_{2}$ and thus allows coarse adjustment of the cooling power by variation of the diameter. Typical cooling rates are in the order of $1.5 \mathrm{~W}$. The temperature of the cold fingers is measured by a PT1000 sensor and regulated to the desired operation temperature via ohmic heating (maximum heating power $10 \mathrm{~W}$ ). The stability of the temperature control loop is $\approx 0.003 \mathrm{~K}$. Three individual temperature-controlled cold stations, for operation in the range between 80 and $150 \mathrm{~K}$, are available for up to three lead-salt diode lasers. An additional cold station, operated near $77 \mathrm{~K}$, houses two mercury cadmium telluride (MCT) photovoltaic detectors.

Each divergent laser beam is collected by a $26^{\circ}$ off-axis ellipsoid (OAE) with 40-mm and $140-\mathrm{mm}$ focal distances. The laser is located at the nearer focal position providing a $f / 2$ aperture and is magnified by a factor 3.5 at the second focal point. The OAE is mounted together with two flat mirrors to

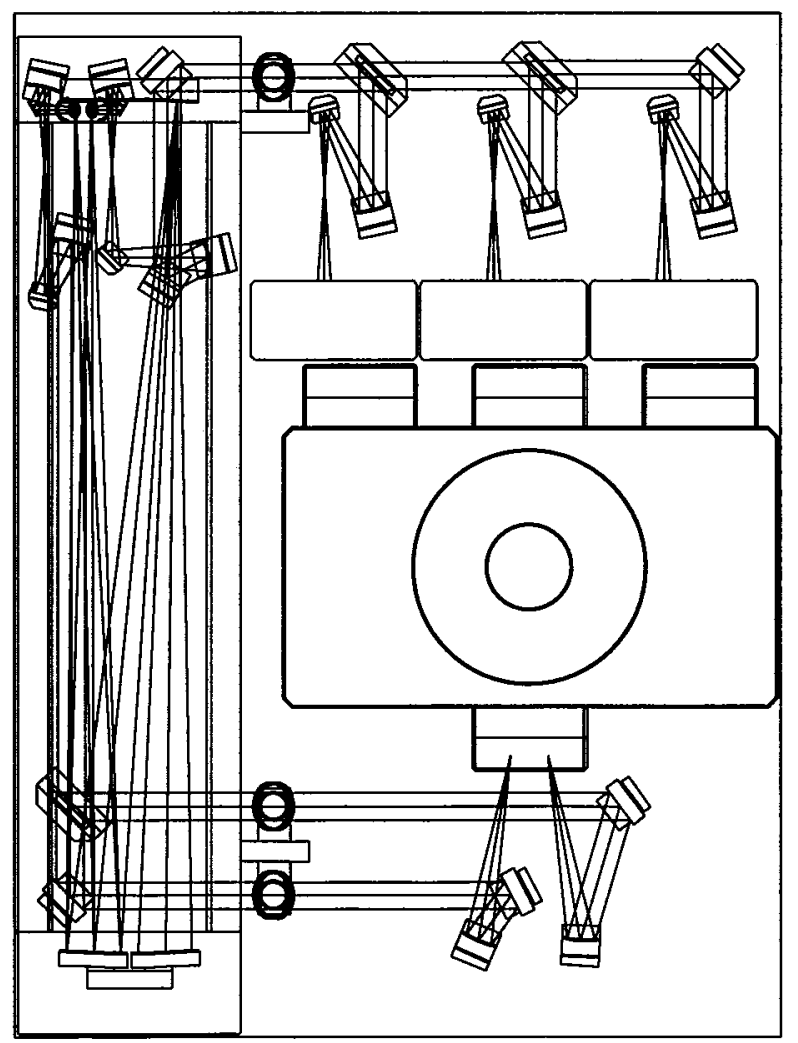

Fig. 1. Schematic of the optical setup of the TRISTAR TDLAS form a mirror objective which is adjustable in three orthogonal axes. Next, a $26^{\circ}$ off-axis parabolic mirror (OAP) collimates the beam into a 14-mm-diameter parallel beam. The intermediate focus serves as a fixed point in the laser spectrometer for alignment purposes. Starting from this point the complete optical setup can be aligned by locating a point light source (for example, a pinhole illuminated by a visible diode laser coupled to an optical fiber) at the focal point. The magnified laser image available at the intermediate focal point allows for pre-alignment of the diode laser by visual inspection of the junction, by viewing the focal point under further magnification through an eyepiece equipped with crosshairs. In addition, a special compact monochromator can be located at the focal point for wavelength determination and investigation of the mode structure of the laser. A detailed description of the mirror objective. alignment tools and the monochromator is given in [14].

Optical multiplexing of the three lasers is achieved by $\mathrm{CaF}_{2}$ or dichroic beam splitters. The beam is coupled into a $0.5-\mathrm{m}$ base length White cell by a combination of a $23^{\circ}$ OAP followed by a $23^{\circ} \mathrm{OAE}$, reversing the combination used for laser collimation, and again providing an intermediate focus for alignment purposes. The cell itself is a specially designed double-corner-cube White cell $[5,12]$ with a volume of approximately 2.71 , providing a maximum optical path length of $64 \mathrm{~m}$ at 128 passes (gold-coated mirrors, reflectivity $>99 \%$ ). After exiting the White cell the beam is re-collimated by a OAE/OAP mirror combination similar to the one used at the cell entrance. After the cell, the beam is split at a $\mathrm{CaF}_{2}$ beam splitter into a reference and a signal part. While the signal beam is focussed onto the signal detector via a $23^{\circ} \mathrm{OAP}$ the reference beam is first transmitted through a short $(5 \mathrm{~cm})$ single-pass cell before it is focussed onto the reference detector. The cell contains the target gases in high concentrations for line identification and accurate line locking. For reactive gases, more than one cell, placed in series, is necessary.

\section{Electronic setup and data acquisition}

A desirable feature for airborne trace-gas detection is the ability to perform measurements with high temporal resolution, since the aircraft travels at speeds of the order of $50-200 \mathrm{~m} / \mathrm{s}$. As for most TDLAS systems, the ultimate time resolution of TRISTAR is limited by the gas exchange time of the White cell, which is in turn limited by the weight and power available for a pump aboard the aircraft. For our cell the resolution time is $\approx 1 \mathrm{~s}$ at a pumping speed of $61 / \mathrm{min}$ (STP). In order to allow high-precision measurements on a $1 \mathrm{~Hz}$ frequency base special measures have to be taken to gain a high signal-to-noise ratio. Significant improvements in sensitivity, in particular for TDLAS applications demanding high temporal resolution, have been achieved by the introduction of high-frequency modulation schemes $[15,16]$. In general, modulation of the laser emission frequency and subsequent phase-sensitive detection promises higher sensitivity than direct measurement of changes in transmitted laser power because the detection frequency is shifted to a regime with reduced laser noise. Because there is a strong $1 / f$ component in the laser noise [17.18]. high-frequency modula- 
tion and detection schemes offer higher sensitivity than conventional wavelength modulation schemes which operate at kHz-modulation frequencies [16]. Therefore a two-tone frequency modulation (TTFM) scheme [19] has been selected for TRISTAR. The electronic setup for TRISTAR, which is shown in Fig. 2, is similar to the one described in detail by Wienhold et al. [15] for an earlier version of a two-laser TDLAS. It consists of a laser modulation module that provides rf-current components at 102 and $122 \mathrm{MHz}$, which are added to the laser dc-currents via bias-Ts, and two demodulation modules for phase-sensitive detection at the difference frequency $(20 \mathrm{MHz})$ for the signal and the reference channels. These compact modules are integrated into the laser control unit (Profile). Preset values of modulation frequency and index, signal attenuation. and local oscillator phase for up to four lasers can be programmed via a fast synchronous serial communication link. A microcomputer based on the AT\&T DSP 32C digital signal processor (DSP) controls the instrument and performs real-time data analysis. Four analog input signals - signal and reference channel in direct and two-tone frequency modulation mode - are read at $100 \mathrm{kHz}$ rate by the DSP. Figure 3 illustrates the data acquisition timing sequence used for TRISTAR. Whereas the time multiplexing for the two laser instrument described by Wienhold et al. [15] relied on consecutive blocking of the individual laser beams by a mechanical chopper, time multiplexing for TRISTAR is achieved by pulsed-current operation of the three lasers.
A current pulse of approximately $2 \mathrm{~ms}$ duration causes the emission of one of the three lasers to sweep across the absorption feature of the target molecule. After a delay of $100 \mu \mathrm{s}$, the next laser is pulsed while the spectra obtained during the previous pulse are processed. A simple rectangular waveform of the laser pulse drive current has some serious drawbacks, in particular relating to multi-mode operation and nonlinear tuning characteristics of the laser during the pulse. We have found that these effects can be minimized by modifying the shape of the current pulse as illustrated in Fig. 3. The pulse is set to initially overshoot the desired operating point and is then reduced after approximately $0.5 \mathrm{~ms}$, at which time an additional current ramp is applied to linearize the tuning of the laser across the absorption feature in $1-5 \mathrm{~ms}$. As described above a rf-current component is added to allow increased sensitivity via TTFM detection. The TTFM signals are used for the data analysis, whereas the laser power in the signal and reference channels are evaluated using the direct-mode signals. After a software correction for any shifts of the line center position, calculated from the reference channel, the spectra are averaged until the DSP receives a request from the host computer to start a new measurement sequence, usually at 1-s intervals. The signal TTFM spectrum is smoothed by a simplified least-squares procedure [20], then a prerecorded background spectrum may be subtracted and a prerecorded calibration spectrum together with a second-order polynomial is fitted to the signal spectrum to yield the trace-gas concen-

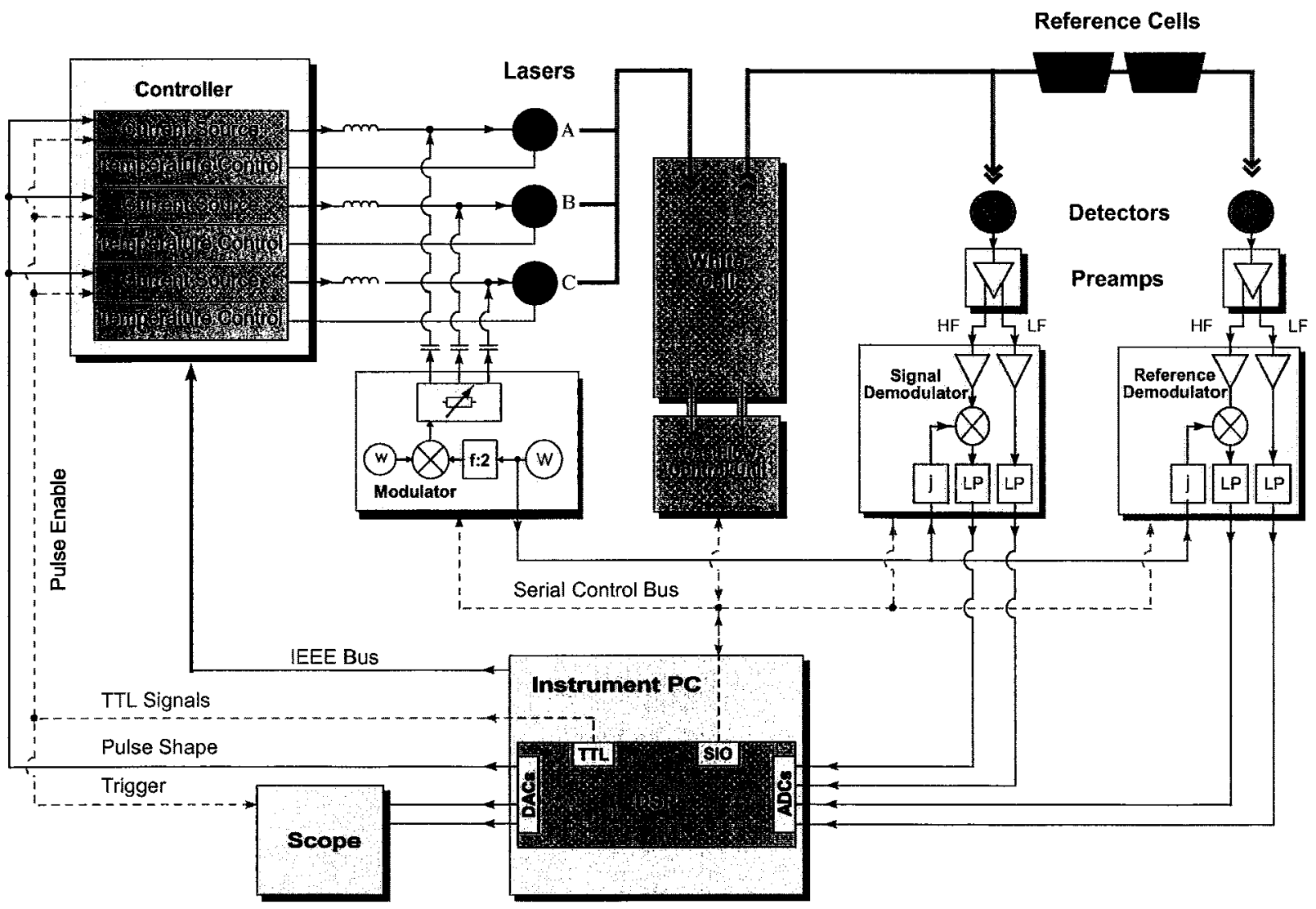

Fig. 2. Schematic of the TRISTAR electronic setup 


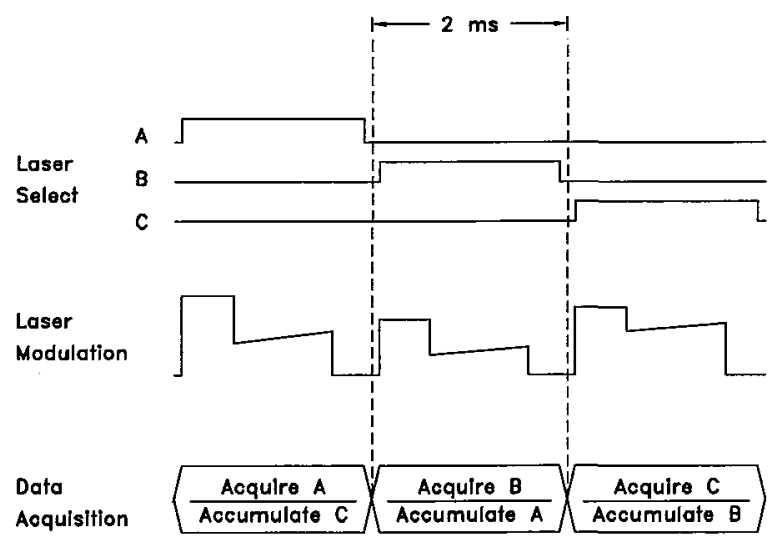

Time

Fig. 3. Data acquisition timing diagram

tration and its error estimate. A subsequent interrupt from the DSP to the PC initiates transfer of the processed data.

For each measurement, the trace-gas concentration, its error estimate, the laser power, and the current pulse parameters are written to the hard disk of the host computer together with diagnostic data such as cell pressure, cell flow, calibration flow, and intake gas temperature. To reduce hard-disk access during flight and to reduce storage requirements, only the calibration, background, and average of ten smoothed ambient spectra are stored in full, with only the results of the fitting procedure and the diagnostics being stored for the remaining ambient spectra.

\section{Gas flow and calibration concept}

Ambient air is sampled with an inlet system designed by the University of Bonn. In Fig. 4 a schematic representation of the inlet system and the gas flow concept is shown. The system is designed to allow future measurements of polar gases such as $\mathrm{HCl}, \mathrm{H}_{2} \mathrm{O}_{2}$, and $\mathrm{HNO}_{3}$. Ambient air is sampled outside the aircraft at a distance of approximately $15 \mathrm{~cm}$ above the fuselage in a rearward direction to avoid aerosol sampling. The gas is pumped through $1 / 2^{\prime \prime}$ PFA tubing and the White cell by a rotary pump (Busch R5 $0016 \mathrm{~B}, 16 \mathrm{~m}^{3} / \mathrm{h}$ ). The cell pressure is regulated to approximately $40 \mathrm{hPa}$ using a feedback loop to adjust a critical orifice (ASCO proportional solenoid valve) in front of the White cell. Mass flow is approximately $61 / \mathrm{min}$ (STP), resulting in a gas exchange time of $\approx 1 \mathrm{~s}$ for the White cell (2.7 l). In-flight calibrations are performed every 10 to $20 \mathrm{~min}$ depending on flight conditions, by replacing ambient air at the tip of the inlet system with a surplus flow of a known calibration gas standard. Calibration gas standards are taken either from tanks (for example for $\mathrm{CO}, \mathrm{N}_{2} \mathrm{O}, \mathrm{CH}_{4}$ ) or permeation devices (for example for $\mathrm{H}_{2} \mathrm{O}_{2}, \mathrm{HNO}_{3}, \mathrm{HCl}$ ). For reactive species, and species that exhibit high wall losses, the calibration line made of PFA tubing can be preconditioned by a permanent purge gas flow via a calibration by-pass. For calibration purposes. a valve in the by-pass is closed and the calibration gas is added to the sampling line. This procedure permits in-flight calibrations of the

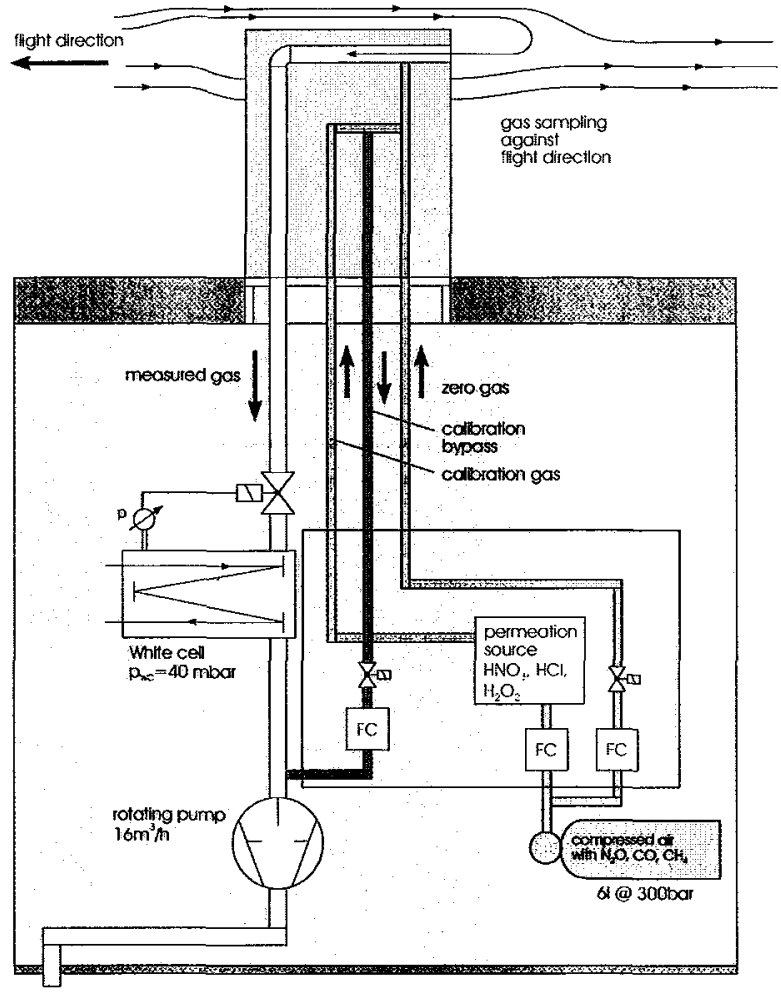

Fig. 4. Schematic of the inlet system, gas flow, and calibration devices

complete system and accounts for first-order losses of reactive or sticky species in the system.

\section{Post-flight data processing}

To assess data quality after the flight, each calibration spectrum is fitted to its successor and instrumental drift in responsivity is quantified by the deviation of the fit result from unity. Correlating this deviation with the corresponding deviation in the measured laser power reveals if power changes caused the drift. Power changes may arise from minute alterations in the optical alignment, due for example to temperature changes in the cabin, or, in certain cases, to changes in the composition of cabin air, which changes broad-band absorptions outside the White cell. A clear correlation between sensitivity and measured laser power will only arise if the laser operates on a single mode, which is desirable but not always achievable with available lasers. In the case of single-mode laser operation, the data quality can be improved by appropriate normalization of the raw data to the laser power. For multimode operation a drift in sensitivity between two successive calibrations is not necessarily correlated with the power signal. In the mode competition case the drift appears to be of stochastic origin, and a linear time interpolation for the instrument response between two successive calibrations is carried out. Typically these corrections are smaller than $2 \%$, which is the precision that can be quoted for the measurement data.

During the POLSTAR II campaign, which took place from Kiruna in Sweden in January 1998, additional correc- 
tions were made based on the intensity of the reference spectral signal. Under the assumptions that the optical density in the reference cell is much larger than the optical density in the White cell and that the reference cell concentration is constant, fluctuations in the reference signal will correspond to changes in the single-mode power of the laser. Since the beamsplitter separating signal and reference channel are placed after the White cell, changes in alignment or in mode competition should affect both channels equally.

\section{Field deployment}

So far TRISTAR has been used during three airborne measurement campaigns in the Arctic lower stratosphere in 1997 and 1998. During the POLSTAR I (Polar stratospheric aerosol experiment) mission in January 1997 and during the POLSTAR II campaign one year later, the instrument was operated on board the research aircraft Falcon 20 from Kiruna airport, Sweden. In March 1997 an additional campaign was performed from the same location using the Dutch Cessna Citation II as part of the STREAM (stratosphere troposphere experiments by aircraft measurements) project. In 1997 TRISTAR was operated in a two-channel mode for CO and $\mathrm{N}_{2} \mathrm{O}$. A third channel devoted to $\mathrm{CH}_{4}$ was added in 1998 . $\mathrm{CO}$ and $\mathrm{N}_{2} \mathrm{O}$ measurements were performed using several absorption lines in the 2150 to $2200 \mathrm{~cm}^{-1}$ spectral region, and for $\mathrm{CH}_{4}$ a feature at $2954.73 \mathrm{~cm}^{-1}$ was selected. The individual absorption lines were selected from the HITRAN data base [21] to avoid cross-sensitivities to other gaseous species in ambient air. In addition, checks for cross-sensitivities were performed by inserting single-pass absorption cells containing high concentrations of potentially interfering gases into the optical path.

Figure 5 shows the time series of $\mathrm{N}_{2} \mathrm{O}$ (b: raw and c: revised data) and diagnostic data (a: flight altitude, d: error of the least-square fit, e: laser power on the signal detector and, f: laser current) obtained during a POLSTAR measurement flight on $30 / 01 / 97$. The objective of this flight was to study the large-scale distribution of trace gases in the lower Arctic stratosphere between Kiruna. Sweden $\left(67.8^{\circ} \mathrm{N}, 20.3^{\circ} \mathrm{E}\right)$ and Spitsbergen, Norway $\left(80.5^{\circ} \mathrm{N}, 21.3^{\circ} \mathrm{E}\right)$. During the first half of the flight the aircraft cruised at $11.8 \mathrm{~km}$ in the lower stratosphere, followed by a descending profile into the troposphere $(<6.3 \mathrm{~km})$ over Spitsbergen. The return flight to Kiruna followed the outward route but at a slightly higher altitude in the stratosphere $(12.5 \mathrm{~km})$ (Fig. $5 \mathrm{a})$. Nitrous oxide was measured using an absorption line at $2103.749 \mathrm{~cm}^{-1}$, at a pressure of $42.5 \mathrm{hPa}$ in the White cell and a flow of approximately $61 / \mathrm{min}$ (STP). Figure $5 \mathrm{c}$ shows the post-processed $\mathrm{N}_{2} \mathrm{O}$ data. As one might expect, $\mathrm{N}_{2} \mathrm{O}$ mixing ratios are constant in the troposphere $(311.8 \pm 4.3 \mathrm{ppbv})$ and decrease above the tropopause to a minimum value of $273 \mathrm{ppbv}$ at the highest flight level in the lower stratosphere. The revised data have been deduced from the raw data, which are shown in Fig. 5 b. The main feature of this revision is the de-trending of the data by interpolation between the regular in-flight calibrations (19 calibrations were performed on this flight), which can be identified in Fig. $5 b$ as spikes due to standard addition (311 6 ppv). Drifts of the instrument between two calibrations can be due to a change of the laser power (Fig. 5e), for example, due to changes in the optical alignment, or changes of the op-

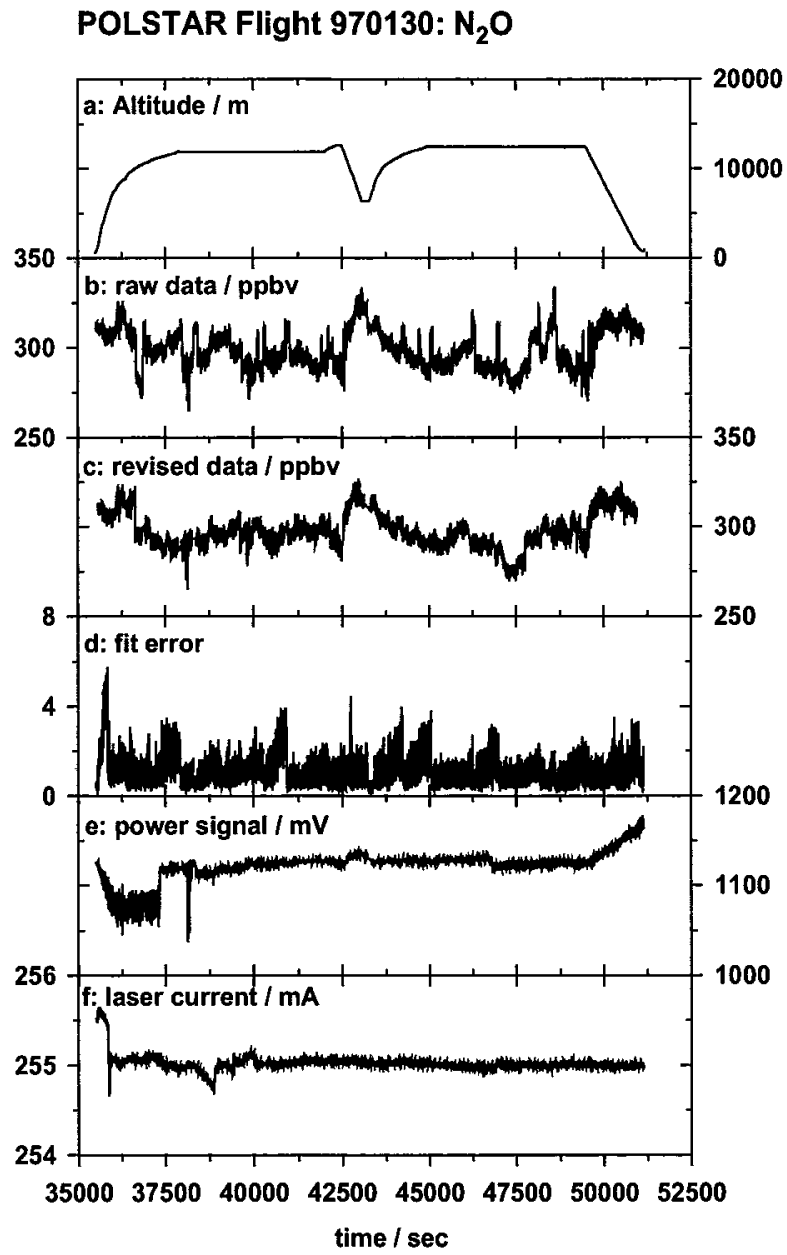

Fig. 5a-f. Time series of $\mathrm{N}_{2} \mathrm{O}$ (b: raw and $\mathbf{c}$ : revised data) and diagnostic data (a: flight altitude, d: error of the least-square fit, e: laser power on the signal detector, and $\mathbf{f}$ : laser current) obtained during a POLSTAR measurement flight on 30/01/97

erational conditions of the laser, in particular changes of its injection current due to the line-locking procedure (Fig. $5 \mathrm{f}$ ). These drifts determine the stability of the instrument. As a measure for the instrument stability the error of the leastsquare line fit can be used, which is mainly determined by changes in the line shape due to instrument drifts or optical interferences (etalons). During this particular flight the fit error varies between 2 and 4 ppbv (Fig. $5 \mathrm{~d}$ ).

An example demonstrating the usefulness of the diagnostics in identifying problematic data is given in the first part of this flight between 35000 and $36000 \mathrm{~s}$. Large fit errors in combination with strong variations of the laser power and a jump in the scan current occurred in the first part of the flight after take-off from Kiruna before the aircraft reached its cruise level. This leads to a structure in the apparent $\mathrm{N}_{2} \mathrm{O}$ in the troposphere during the ascent, even after post-processing of the data (Fig. 5c). The diagnostic data, in particular the scan laser current, indicate that the cold-station temperature was drifting, so that the data should be considered as unreliable. 
The precision of the measurement is further limited by noise. From Fig. $5 \mathrm{c}$ one can estimate a noise band in the order of $\approx 6 \mathrm{ppbv}$ (peak to peak) for the revised data recorded at $1-\mathrm{s}$ time resolution. Whereas improvements in the precision can be gained from longer integration times, de-trending of instrument drifts is accomplished by interpolation between in-flight calibrations. Figure 6 shows a contour plot of the 19 calibration spectra versus spectrum channel number obtained during the flight (upper panel), indicating that the position (channel number) of the line and its shape do not change very much during the flight, which is expected for an accurate linelocking procedure. On the other hand, the amplitude of the calibration signal and the power of the laser for the calibration spectrum varied significantly during the flight $(+/-5 \%)$. The lower panel of Fig. 6 shows the relative changes of the calibration signal amplitude (open circles), the laser power during calibration (open squares), and the calibration signal normalized to the calibration power (filled circles). Clearly, in this case, the calibration signal amplitude (system sensitivity) and measured laser power are not correlated. Therefore normalization of the signals to the power cannot be used for de-trending the data. Instead, a linear interpolation between two calibrations was used for post-flight analysis.

A contrary case of a power-correlated drift of the calibration signal is shown in Figs. 7 and 8 for data obtained on a measurement flight during the STREAM campaign on $25 / 03 / 97$. The purpose of this flight was to obtain a latitudinal cross section during the transfer fight from Kiruna to Amsterdam (The Netherlands) at a constant altitude of approximately $11.8 \mathrm{~km}$ (Fig. 7a). Most of the flight took place in the tropopause region, so that only small variations of $\mathrm{N}_{2} \mathrm{O}$ are expected. The raw data (Fig. 7b) exhibit significant

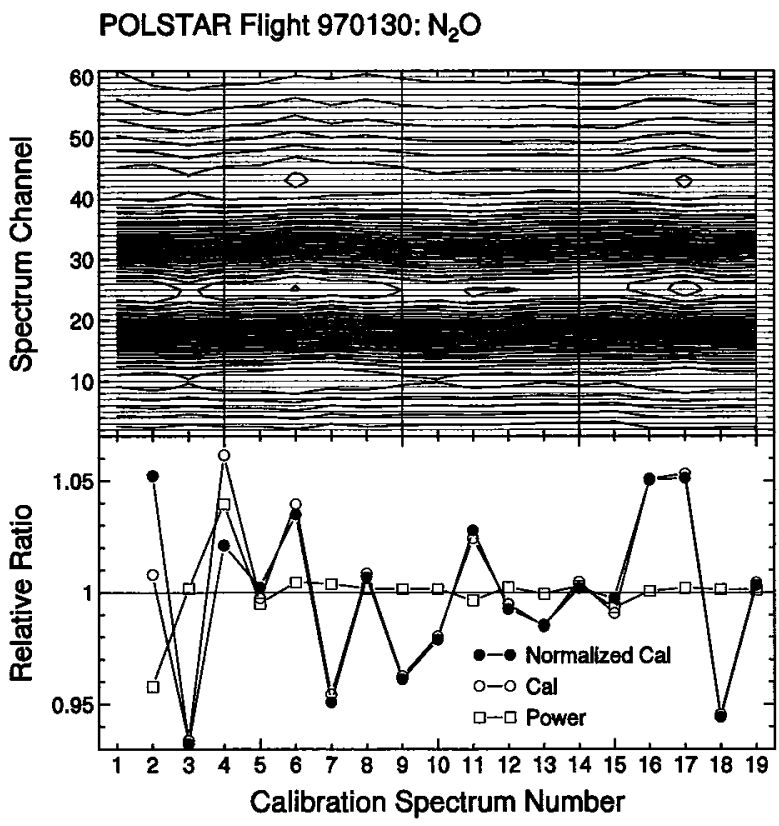

Fig. 6. Contour plot of the 19 calibration spectra versus spectrum channel number obtained during the flight on 30/01/97 (upper panel). The lower panel shows the relative changes of the calibration signal amplitude (open circles), the laser power during calibration (open squares), and the calibration signal normalized to the calibration power (filled circles)
STREAM Flight 970325: $\mathrm{N}_{2} \mathrm{O}$

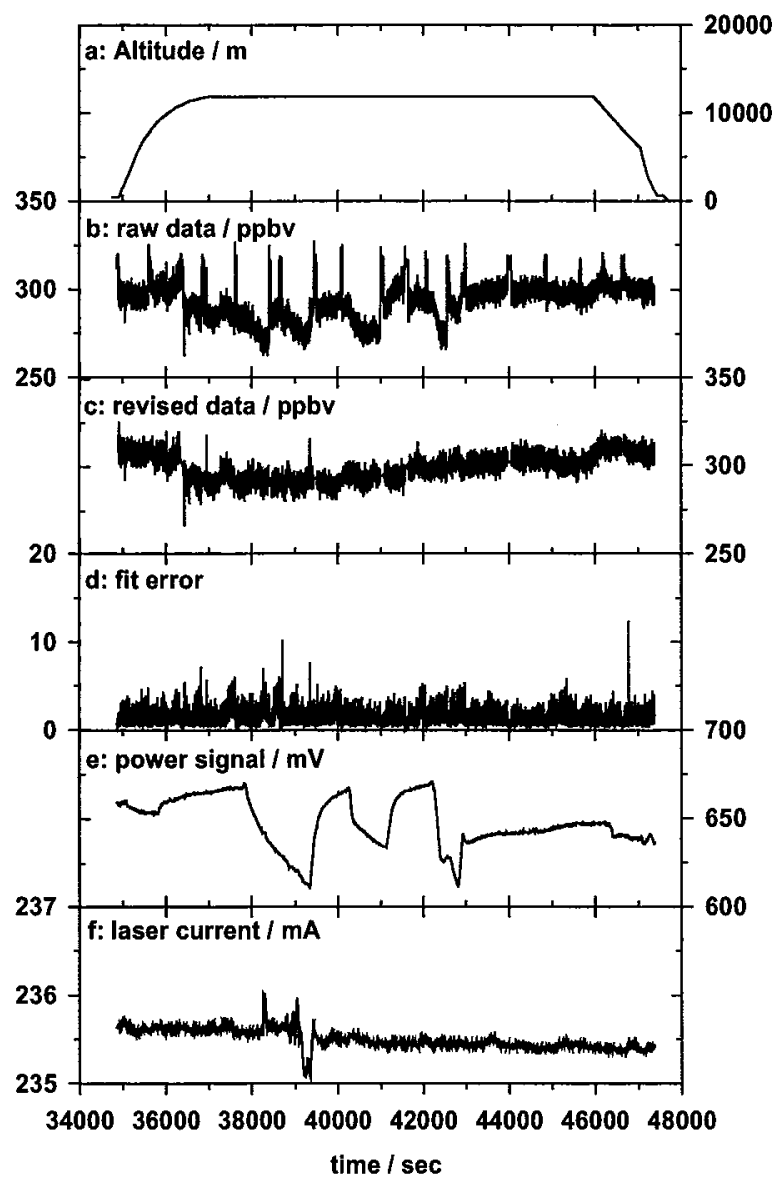

Fig. 7a-f. Time series of $\mathrm{N}_{2} \mathrm{O}$ (b: raw and $\mathbf{c}$ : revised data) and diagnostic data (a: flight altitude, d: error of the least-square fit, e: laser power on the signal detector, and $\mathbf{f}$ : laser current) obtained during a STREAM measurement flight on 25/03/97

deviations in the $\mathrm{N}_{2} \mathrm{O}$ mixing ratios just before and after a calibration. The corresponding drifts are correlated with strong variations in the laser power (Fig. 7e). Therefore normalization of the calibration signal to the signal power reduces the inter-calibration variability significantly (Fig. 8. lower panel), allowing a de-trending of the data using a power normalization. The results of this post-processing are shown in Fig. $7 \mathrm{c}$.

The different behavior during these two flights is due to the emission characteristics of the laser. A strong correlation between laser power and signal size can be expected only for single-mode operation of the laser, which was achieved for the flight on $25 / 03 / 97$. If the laser is not single mode (30/01/97), mode competition can mask the power dependency of the signal strength. Unfortunately, single-mode performance is not always available, nor can it be guaranteed that the mode characteristics of individual lasers will remain constant over periods spanning measurement campaigns, especially if it is not possible to continuously maintain a cryogenic laser environment.

On average, the reproducibility of the calibrations during all three campaigns for $\mathrm{CO}, \mathrm{N}_{2} \mathrm{O}$ and $\mathrm{CH}_{4}$ is of the order of $\pm 2 \%(3 \sigma)$ and the calibration accuracy is $\pm 2.8 \%$. The pre- 
STREAM Flight 970325: $\mathrm{N}_{2} \mathrm{O}$

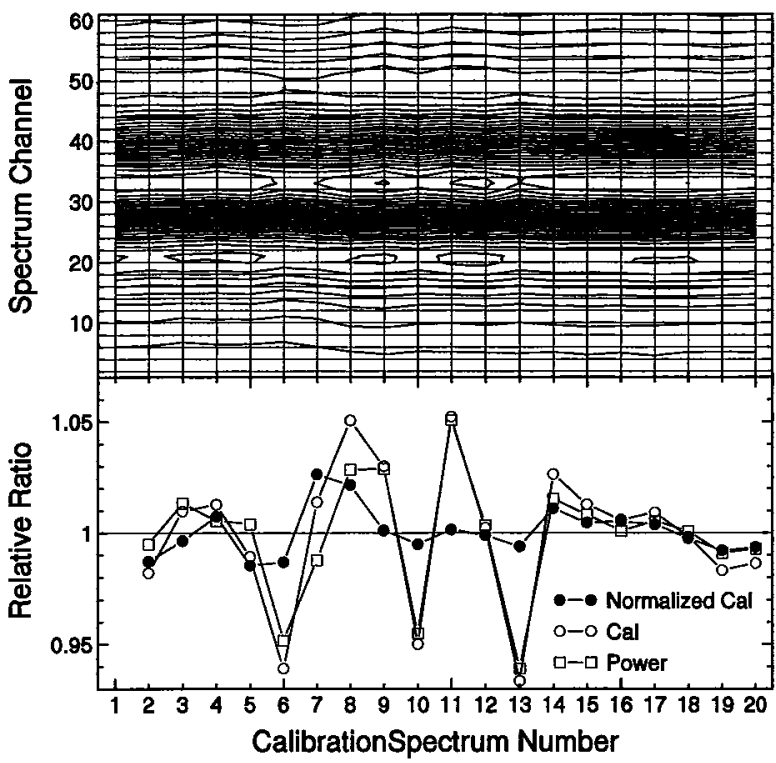

Fig. 8. Contour plot of the 20 calibration spectra versus spectrum channel number obtained during the flight on 25/03/97 (upper panel). The lower panel shows the relative changes of the calibration signal amplitude (open circles), the laser power during calibration (open squares), and the calibration signal normalized to the calibration power (filled circles)

cision strongly depends on the noise level, and therefore on the properties and operating conditions of the lasers. Typical noise levels $(3 \sigma)$ for $\mathrm{N}_{2} \mathrm{O}, \mathrm{CO}$, and $\mathrm{CH}_{4}$ at 1 -s resolution are $\leq 10 \mathrm{ppbv}, \leq 20 \mathrm{ppbv}$, and $\leq 30 \mathrm{ppbv}$, respectively.

\section{Summary}

A three-laser TDLAS system called TRISTAR has been developed for airborne measurements of $\mathrm{N}_{2} \mathrm{O}, \mathrm{CO}$, and $\mathrm{CH}_{4}$ in the tropopause region and successfully used during three Arctic winter campaigns in 1997 and 1998 on two different platforms, a Falcon 20 and a Cessna Citation II. The instrument uses a newly developed combination of pulsed-current operation and two-tone frequency modulation, which permits time-multiplexed operation of three individual lasers with high sensitivity. TRISTAR allows fast (1-s time resolution), precise (low percent range and low ppbv range), and accurate (low percent range) measurements of the above species. The overall performance of the system and its ability to perform in-flight calibrations of the inlet system and sensor will allow us to extend our measurement capabilities further to more reactive species (for example $\mathrm{HNO}_{3}, \mathrm{H}_{2} \mathrm{O}_{2}, \mathrm{or} \mathrm{HCl}$ ) in the near future.

Acknowledgements. This work was supported by the German Ministry of Research and Education (BMBF) and the Comission of the European Countries (DG-XII)

\section{References}

1. H.I. Schiff, G.I. Mackay, J. Bechera: In Air Monitoring by Spectroscopic Techniques, ed by M.W. Sigrist (Wiley. New York 1994) pp. 239-333

2. H. Fischer, P. Bergamaschi, F.G. Wienhold, T. Zenker, G.W. Harris: SPIE Proc. 2834, 130 (1996)

3. A. Fried, S. Sewell, B. Henry, B.P. Wert, T. Gilpin, J.R. Drummond J. Geophys. Res. 102, 6253 (1997)

4. G.W. Harris, D. Klemp, T. Zenker, J.P. Burrows, B. Matthieu: J. Atmos. Chem. 15, 315 (1992)

5. J. Roths, T. Zenker, U. Parchatka, F. Wienhold, G.W. Harris: Appl Opt. 35, 7075 (1996)

6. G.W. Sachse, G.F. Hill, L.O. Wade, M.G. Perry: J. Geophys. Res. 92. 2071 (1987)

7. I. Podolske, M. Loewenstein: Appl. Opt 32, 5324 (1993)

8. C.R. Webster, R.D. May, C.A. Trimble, R.G. Chave, J. Kendall: Appl. Opt. 33, 454 (1994)

9. F.G. Wienhold, H. Fischer, G.W. Harris: Infrared Phys. Techn. 37, 67 (1996)

10. K.J. Hargraves, U.M. Skiba, D. Fowler, J.R.M. Arah, F.G. Wienhold, L. Klemendtsson, B. Galle: J. Geophys. Res. 99, 16569 (1994)

11. P. Bergamaschi, M. Schupp, G.W. Harris: Appl. Opt. 33, 7704 (1994)

12. J.U. White: J. Opt. Soc. Am. 66, 411 (1976)

13. W.J. Riedel, M. Knothe: Deutsches Patent No. 4033383 (1993)

14. W.J. Riedel: SPIE Proc. 1433, 179 (1991)

15. F.G. Wienhold, T. Zenker, G.W. Harris: SPIE Proc 2112, 31 (1994)

16. P. Werle: SPIE Proc. 2092, 4 (1992)

17. H. Fischer, H. Wolf, B. Halford, M. Tacke: Infrared Phys. 31, 381 (1991)

18. H. Fischer, M. Tacke: J. Opt. Soc. Am. B 8, 1824 (1991)

19. D.E. Cooper, R.E. Warren: J. Opt. Soc. Am. B 4, 470 (1987)

20. A. Savitzky, M.J.E. Golay: Anal. Chem. 36, 1627 (1964)

21. L.S. Rothman, R.R. Gamache, R.H. Tipping, C.P. Rinsland, M.A.H. Smith, D.C. Brenner, V.M. Devi, J-M. Flaud, C Camy-Peyret A. Perrin, A. Goldman, S.T. Massie, L.R. Brown, R.A. Toth: I. Quant. Spectrosc. Radiat. Transfer 48, 469 (1992) 